An Extended Abstract Submitted to $43^{\text {rd }}$ AIAA Thermophysics Conference, New Orleans, June 2012

\title{
Electron Stark Broadening Database for Atomic N, O, and C Lines
}

\author{
Yen Liu ${ }^{1}$, Winifred M. Huo ${ }^{2}$, Alan Wray ${ }^{3}$, and Duane F. Carbon ${ }^{4}$ \\ NASA Ames Research Center, Moffett Field, CA, 94035, USA
}

\begin{abstract}
A database for efficiently computing the electron Stark broadening line widths for atomic $\mathrm{N}, \mathrm{O}$, and $\mathrm{C}$ lines is constructed. The line width is expressed in terms of the electron number density and electronatom scattering cross sections based on the Baranger impact theory. The state-to-state cross sections are computed using the semiclassical approximation, in which the atom is treated quantum mechanically whereas the motion of the free electron follows a classical trajectory. These state-to-state cross sections are calculated based on newly compiled line lists. Each atomic line list consists of a careful merger of NIST, Vanderbilt, and TOPbase line datasets from wavelength $50 \mathrm{~nm}$ to $50 \mu \mathrm{m}$ covering the VUV to IR spectral regions. There are over 10,000 lines in each atomic line list. The widths for each line are computed at 13 electron temperatures between $1,000 \mathrm{~K}-50,000 \mathrm{~K}$. A linear least squares method using a four-term fractional power series is then employed to obtain an analytical fit for each line-width variation as a function of the electron temperature. The maximum $\mathrm{L}_{2}$ error of the analytic fits for all lines in our line lists is about $5 \%$.
\end{abstract}

\section{Introduction}

$\mathrm{T}_{\mathrm{b}}^{\mathrm{H}}$ HE emission and absorption coefficients in the radiative transfer equation for a bound-bound transition depend on four quantities: the line position, the line oscillator strength or the Einstein coefficients, the line shape function, and energy state populations. The first two quantities are intrinsic properties of atoms and molecules. The NIST atomic spectra database [1], the Vanderbilt University (now also NIST) MCHF/MCDHF

\footnotetext{
${ }^{1}$ Research Scientist, yen.liu@nasa.gov

${ }^{2}$ Ames Associate, winifred.huo@nasa.gov

${ }^{3}$ Research Scientist, alan.wray@nasa.gov

${ }^{4}$ Research Scientist, duane.f.carbon@nasa.gov
} 
database [2], and the Opacity Project [3] database TOPbase [4-5] provide the most up-todate tabulation of these two quantities for many atomic species. However, their data are not all consistent to each other. For molecular species, on the other hand, most calculations still rely on approximate methods, such as the Dunham expansion for line positions and the R-centroid approximation for Einstein coefficients. The line shape function is determined by natural, Doppler, and collisional (pressure) broadening effects. While the natural and Doppler broadening shape functions are easy to determine, collisional broadening widths and shifts and rate coefficients to compute energy state populations require detailed quantum mechanical calculations of state-to-state cross sections involving the complete spectrum of energy states. Today, the most comprehensive source for collisional plasma (Stark) broadening is Griem's book [6] published 37 years ago. His calculations were actually based on a limited number of states and covered only a small portion of transitions in the NIST line lists. In an effort to support the NASA Hypersonics Project's technology challenges: (1) Develop new tools and technologies to enable high mass planetary entry and (2) Decrease uncertainty in aeroheating prediction by $50 \%$, a new radiation code HyperRad is currently being developed at NASA Ames Research Center. Some of its key features are as follows:

- Physics-based modeling for energy populations using coupled thermal, chemical, and radiative nonequilibria

- Efficient and accurate linelist-driven database

- Careful and complete merger of NIST, Vanderbilt, and TOPbase line datasets; the current database includes $\mathrm{N}, \mathrm{N}^{+}, \mathrm{O}, \mathrm{O}^{+}, \mathrm{C}, \mathrm{C}^{+}[7]$, and is easily extended to other atomic species

- Molecular database based on ab initio calculations for electric dipole and quadrupole, magnetic dipole, and spin-forbidden transitions which include fine structure, pre-dissociation, and non-adiabatic corrections; the current database includes $\mathrm{N}_{2}, \mathrm{O}_{2}, \mathrm{C}_{2}, \mathrm{NO}, \mathrm{CO}, \mathrm{CN}, \mathrm{N}_{2}{ }^{+}, \mathrm{CO}^{+}, \mathrm{C}_{3}$, and $\mathrm{C}_{2} \mathrm{H}$ [8]

- Microscopic collisional state-to-state cross sections currently based on a semiclassical method [9]; more accurate, quantal calculations are planned

- Line broadening widths and shifts due to the Stark effect based on computed cross sections; Line-widths parameterized for efficiency up to $50,000 \mathrm{~K}$; maximum averaged error of parameterization is $5 \%$

- Nonequilibrium energy state populations based on a multi-group maximum entropy method; Macroscopic group equations and rate coefficients derived from moments of the master equations [10]

- Three-dimensional, unstructured-grid approach for radiation transport applicable to any body shape and any CFD code

- Designed ground-up for high-end computing in terms of efficiency and parallelization

In this paper, we present some HyperRad results for the computations of electron Stark broadening widths between $1,000 \mathrm{~K}$ and $50,000 \mathrm{~K}$ for atomic $\mathrm{N}, \mathrm{O}$, and $\mathrm{C}$ lines based on the Baranger impact theory [11-13] and a semi-classical approximation [14] using the complete set of energy levels and line lists compiled in Ref. 7. We also show that the Stark widths can be parameterized for efficiency using an analytical fit. 


\section{Impact Theory and Semi-classical Calculation}

The theory of collisional (pressure) broadening has been extensively studied by Baranger [11-13]. Using the impact approximation, an assumption that collisions take place sequentially and that two strong collisions never occur simultaneously, he showed that the collisional broadening shape function for a light-emitting particle (atom, molecule, or ion) in a gas of perturbers is a Lorentzian. The line shape of an isolated transition from an initial state $i$ to a final state $f$ can be expressed in terms of the halfwidth at half maximum (HWHM) $\gamma_{i f}$ and frequency shift $\delta_{i f}$ as

$$
g_{i f}(\omega)=\frac{1}{\pi} \frac{\gamma_{i f}}{\left(\omega-\omega_{i f}-\delta_{i f}\right)^{2}+\gamma_{i f}^{2}} .
$$

Here

$$
\omega_{i f}=\omega_{i}-\omega_{f}
$$

is positive for emission and negative for absorption. For a complete spectrum, one must sum over all possible final states and average over all initial states and all possible types of motion of the perturbers. The width $\gamma_{i f}$ can then be expressed in terms of the perturber number density $n_{p}$, the perturber velocity distribution function $f(v)$, and scattering cross sections $\sigma$ as

$$
\gamma_{i f}=\frac{n_{p}}{2} \int_{0}^{\infty} v f(v)\left(\sum_{j \neq i} \sigma_{i j}+\sum_{j \neq f} \sigma_{f j}+\sigma_{\text {elas }}\right) d v .
$$

Here $\sigma_{i j}\left(\sigma_{f j}\right)$ is the inelastic cross section from the initial state $i$ (final state $f$ ) to a state $j$, and $\sigma_{\text {elas }}$ is a combination of elastic cross sections $\sigma_{i i}$ and $\sigma_{f f}$, and the cross term. For electron-atom collisions, one of the most successful closed coupling approaches for computing the collisional cross section is the R-matrix with pseudo states (RMPS) method $[15,16]$. However, the RMPS method can only handle a limited number of states, not the complete spectrum of states required in a Stark line shape calculation. We therefore adopt a semi-classical approximation [14] for our initial database construction. In the semi-classical approximation, the atom is treated quantum mechanically whereas the motion of the free electron follows a classical trajectory. The semi-classical inelastic cross section is thus given by

$$
\sigma_{i j}=\int_{0}^{\infty} 2 \pi b d b \frac{1}{2 J_{i}+1} \sum_{m_{i}, m_{j}}\left|\left\langle J_{i} m_{i}|T| J_{j} m_{j}\right\rangle\right|^{2},
$$

and the semi-classical elastic cross sections is expressed as

$$
\begin{array}{r}
\sigma_{\text {elas }}=\int_{0}^{\infty} 2 \pi b d b\left[\frac{1}{2 J_{i}+1} \sum_{m_{i}, m_{i}^{\prime}}\left|\left\langle J_{i} m_{i}^{\prime}|T| J_{i} m_{i}\right\rangle\right|^{2}+\frac{1}{2 J_{f}+1} \sum_{m_{f}, m_{f}^{\prime}}\left|\left\langle J_{f} m_{f}^{\prime}|T| J_{f} m_{f}\right\rangle\right|^{2}\right. \\
-2 \operatorname{Re} \sum_{\substack{m_{i}, m^{\prime} \\
m_{f}, m_{f}^{\prime} \\
M}}(-1)^{2 J_{f}+m_{i}+m_{i}^{\prime}}\left(\begin{array}{ccc}
J_{f} & 1 & J_{i} \\
m_{f}^{\prime} & M & -m_{i}^{\prime}
\end{array}\right)\left(\begin{array}{ccc}
J_{f} & 1 & J_{i} \\
m_{f} & M & -m_{i}
\end{array}\right) \\
\\
\left.\times\left\langle J_{i} m_{i}^{\prime}|T| J_{i} m_{i}\right\rangle\left\langle J_{f} m_{f}^{\prime}\left|T^{*}\right| J_{f} m_{f}\right\rangle\right] .
\end{array}
$$


In these expressions, $b$ designates the classical impact parameter of the perturber and $(J, m)$ is the total angular momentum and its projection on the axis normal to the collision plane. The symbol $T$ denote the transition matrix operator

$$
\langle j|T| k\rangle=\delta_{j k}-\left\langle j\left|\exp \left[-\frac{i}{\hbar} \int_{-\infty}^{\infty} V d t\right]\right| k\right\rangle,
$$

where $V$ is the interaction potential between the radiator and perturber. Collisional broadening in general is referred to as Stark broadening if the perturber is a charged particle. Here we consider electron Stark broadening. Let $\mathbf{r}$ and $\mathbf{r}^{\prime}$ be position vectors of the free electron and atomic electrons, respectively. For electron-atom scattering, $V$ is simply the Coulomb potential

$$
V\left(\mathbf{r}, \mathbf{r}^{\prime}\right)=\frac{Z e^{2}}{r}-\sum_{k=1}^{Z} \frac{e^{2}}{\left|\mathbf{r}-\mathbf{r}_{k}^{\prime}\right|},
$$

where the free electron position $\mathbf{r}$ follows the classical trajectory. The computation of the cross sections in Eqs. (4) and (5) and details of differences from the previous theoretical calculations of Griem [6] and Riviere [17] will be presented in a companion paper [9].

\section{Comparisons with Experimental and Theoretical Data}

For our calculations, we adopt the HyperRad atomic line lists for N, O, and C developed by Carbon and Huo [7]. The lists consist of a careful merger of NIST, Vanderbilt, and TOPbase line datasets from wavelength $50 \mathrm{~nm}$ to $50 \mu \mathrm{m}$ covering the VUV to IR spectral regions. TOPbase lines were converted from $L S$ coupling to $J J$ coupling to include fine structure so that they are consistent with the NIST and Vanderbilt datasets. The line lists contain over 10,000 lines for each species in this spectral range. In Figure 1, we plot the Einstein $A_{i f}$ coefficients for all the $\mathrm{N}$ lines below 5 $\mu \mathrm{m}$ in the line list. In the figure, we also include the NEQAIR [18] line list for comparison. Note that NEQAIR has no data above $4 \mu \mathrm{m}$.

In our calculations, we first compute the state-to-state cross sections in terms of electron velocity or energy, and the data are then convolved with the free electron distribution function to form the state-to-state rate coefficients. Using Eq. (3), we obtain the Stark width for a spectral line by first summing the rate coefficients for all other possible transitions from both the initial and final states of the line. The state-to-state cross sections and rate coefficients are also saved to compute the energy level populations. In order to evaluate the impact theory and the semi-classical approximation, we compare our calculations with several experimental data [19-21], as well as with Griem's theoretical data [6], as shown in Figure 2 for several $N$ lines in the UV region. Our calculations involve a complete set of energy states and therefore show relatively larger values than Griem's data, but most are within the experimental uncertainties. Details of the comparisons, also including $\mathrm{O}$ and $\mathrm{C}$ lines, will be given in the final paper. 


\section{Electron Stark Broadening Database}

As described in section II, the computation of the collisional broadening shape function involves very complicated and time-consuming procedures. Since the broadening mechanism is due to collisions, one must know the velocity distribution function of the perturber to compute the widths and shifts. In general, the distribution function can be expressed as a function of the macroscopic moments if moment equations of the Boltzmann equation are used to describe the fluid motion. Thus, for efficiency, the widths and shifts can be pre-computed and parameterized using macroscopic flow variables. In this paper, we employ the Maxwellian distribution characterized by an electron temperature $T_{e}$ to construct a database for Stark widths under the assumption that the free electrons are in translational equilibrium. The database for Stark shifts will be constructed in the future work. We first compute the widths at $13 T_{e}$ positions, $1,000 \mathrm{~K}, 2,500 \mathrm{~K}, 5,000 \mathrm{~K}, 7,500 \mathrm{~K}, 10,000 \mathrm{~K}, 15,000 \mathrm{~K}, 20,000 \mathrm{~K}, 25,000 \mathrm{~K}, 30,000 \mathrm{~K}$, $35,000 \mathrm{~K}, 40,000 \mathrm{~K}, 45,000 \mathrm{~K}$, and $50,000 \mathrm{~K}$, for every transition line in our atomic $\mathrm{N}, \mathrm{O}$, and $\mathrm{C}$ line lists. Figure 3 shows the temperature variation of the Stark widths for all $\mathrm{N}$ lines. Each line width shown in the figure is normalized by its width at $10,000 \mathrm{~K}$. Due to lack of data, many computer codes employ a universal single temperature extrapolation formula for all lines to obtain widths at temperatures other than the reference one. For the purpose of comparison, such an extrapolation formula used in NEQAIR is also shown in the figure. Figure 3 also shows that the temperature variation of the Stark width is very smooth. This indicates that the line-width variation with $T_{e}$ can be parameterized for efficiency using either a spline or an analytical fit. The former is more accurate, but requires more storage for each line. In this paper, we construct an analytical fit for each line using the linear least squares method. The analytical fit is expressed in terms of basis functions $\phi^{m}\left(T_{e}\right)$ as

$$
\frac{\gamma_{i f}}{n_{e}}\left(T_{e}\right)=\sum_{m} a_{i f}^{m} \phi^{m}\left(T_{e}\right)
$$

For a four-term fractional power series $\phi^{m}=\left(T_{e}\right)^{\frac{1}{m}}, m=2-5$, the maximum $L_{2}$ error for all $\mathrm{N}$ lines is about 5\%. Almost all large errors occur at temperatures below 2,500K, where Stark broadening is not important. At temperatures above $10,000 \mathrm{~K}$, the errors in general are below 1\%. Figure 4 shows that the analytical fits essentially reproduce the semi-classical calculations at $12,500 \mathrm{~K}$ for all $\mathrm{N}$ lines. Results for $\mathrm{O}$ and $\mathrm{C}$ lines will be presented in the final paper.

\section{References}

1. Ralchenko, Y., Kramida, A.E., Reader, J. and NIST ASD Team (2010). NIST Atomic Spectra Database (version 4.0), [Online]. Available: http://physics.nist.gov/asd. National Institute of Standards and Technology, Gaithersburg, MD. 
2. Froese Fischer C. and Tachiev G., MCHF/MCDHF Collection, Version 2, Available online at http://physics.nist.gov/mchf. National Institute of Standards and Technology, Gaithersburg, MD.

3. The Opacity Project Team, 1995, The Opacity Project Vol. 1, Institute of Physics Publications, Bristol, UK

4. Cunto W., Mendoza C., 1992, Rev. Mexicana Astron. Astrofis. 23, 107.

5. Cunto W., Mendoza C., Ochsenbein F., Zeippen C.J., 1993, A\&A 275, L5.

6. Greim, H. R., "Spectral line Broadening by Plasmas," Academic Press, Inc., 1974.

7. Carbon, D.F. and Huo, W., in preparation, 2012.

8. Schwenke, D.W., Chaban, G., and Jaffe, R.L., in preparation, 2012.

9. Huo, W.M., in preparation, 2012.

10. Liu, Y., Vinokur, V., Panesi, M., and Magin, T., AIAA paper 2010-4332, 2010.

11. Baranger, M., "Simplified Quantum-Mechanical Theory of Pressure Broadening," Phys. Rev. 111 (2), 1958.

12. Baranger, M., "Problem of Overlapping Lines in the Theory of Pressure Broadening," Phys. Rev. 111 (2), 1958.

13. Baranger, M., "General Impact Theory of Pressure Broadening," Phys. Rev. 112 (3), 1958.

14. Seaton, M. J., Proc. Phys. Soc., 79, 1105-1117, 1962.

15. Marchalant, P., and Bartschat, K., J. Phys. B, 30, 1997

16. Zatsarinny, O., Bartschat, K., Bandurina, L., and Gedeon, V., Phys. Rev. A, 71, 2005.

17. Rivière, Ph., "Systematic semi-classical calculations of Stark broadening parameters of NI, OI, NII, OII multiplets for modeling the radiative transfer in atmospheric air mixture plasmas", Journal of Quantitive Spectroscopy and Radiative Transfer, 72, 2002.

18. Whiting, E.E., Park, C., Liu, Y., Arnold, J.O., and Paterson, J.A., "NEQAIR96, Nonequilibrium and Equilibrium Radiative Transport and Spectra Program: Users Manual," Technical Report NASA RP-1389, Ames Research Center, Moffett Field, CA, 1996

19. Jones, D.W., Pichler, G., and Wiese, W. L., Phys. Rev. A 35, 1987.

20. Nubbemeyer, H., Phys. Rev. A 22, 1980.

21. Culmann, E., and Labuhn, F., JQSRT 20, 1978. 


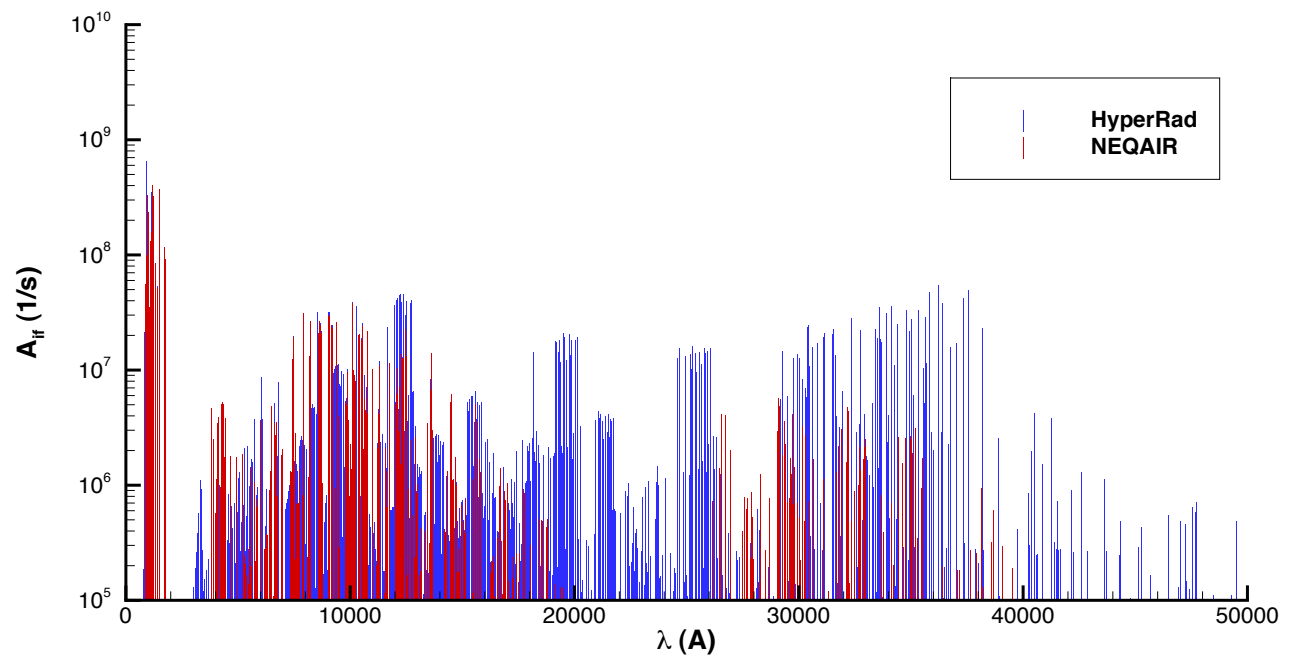

Figure 1. Atomic N lines

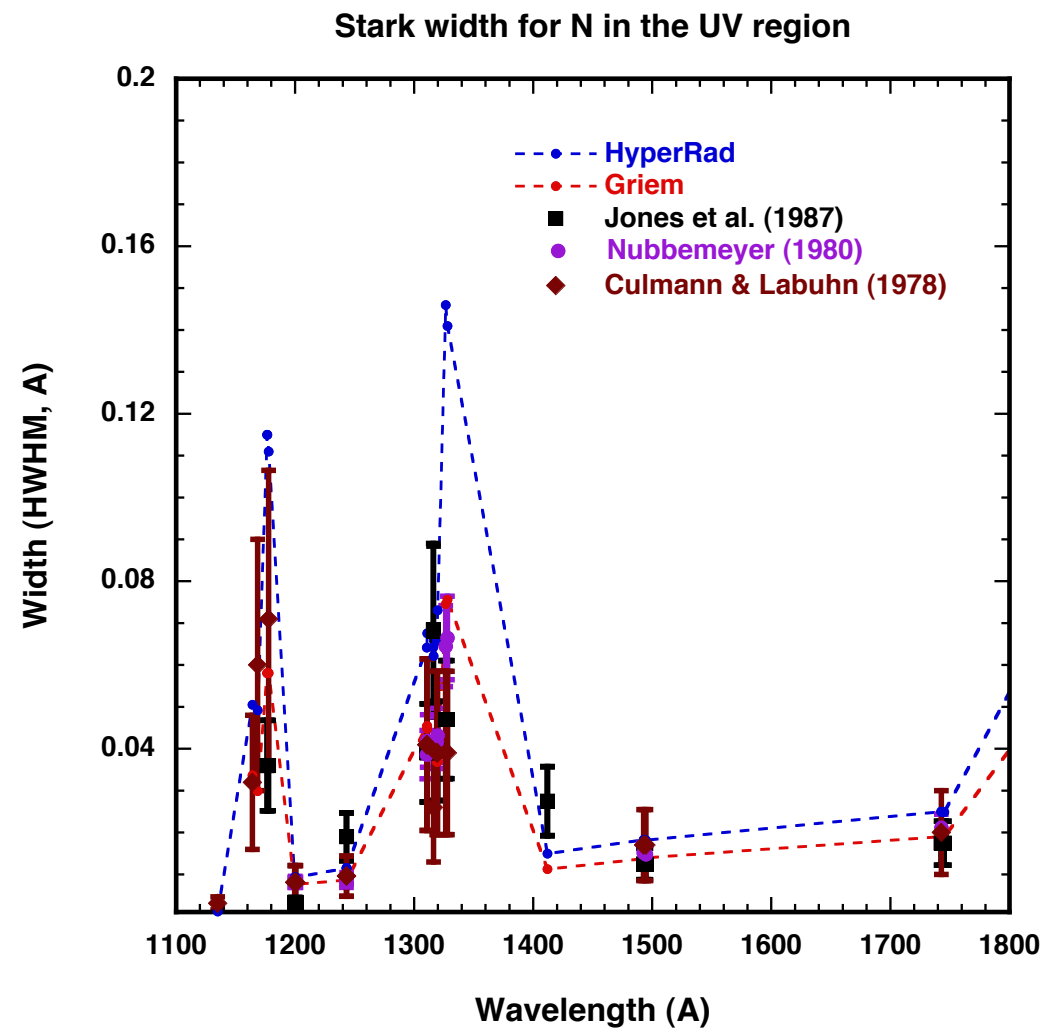

Figure 2. Comparison of Stark width with experimental and theoretical data. 


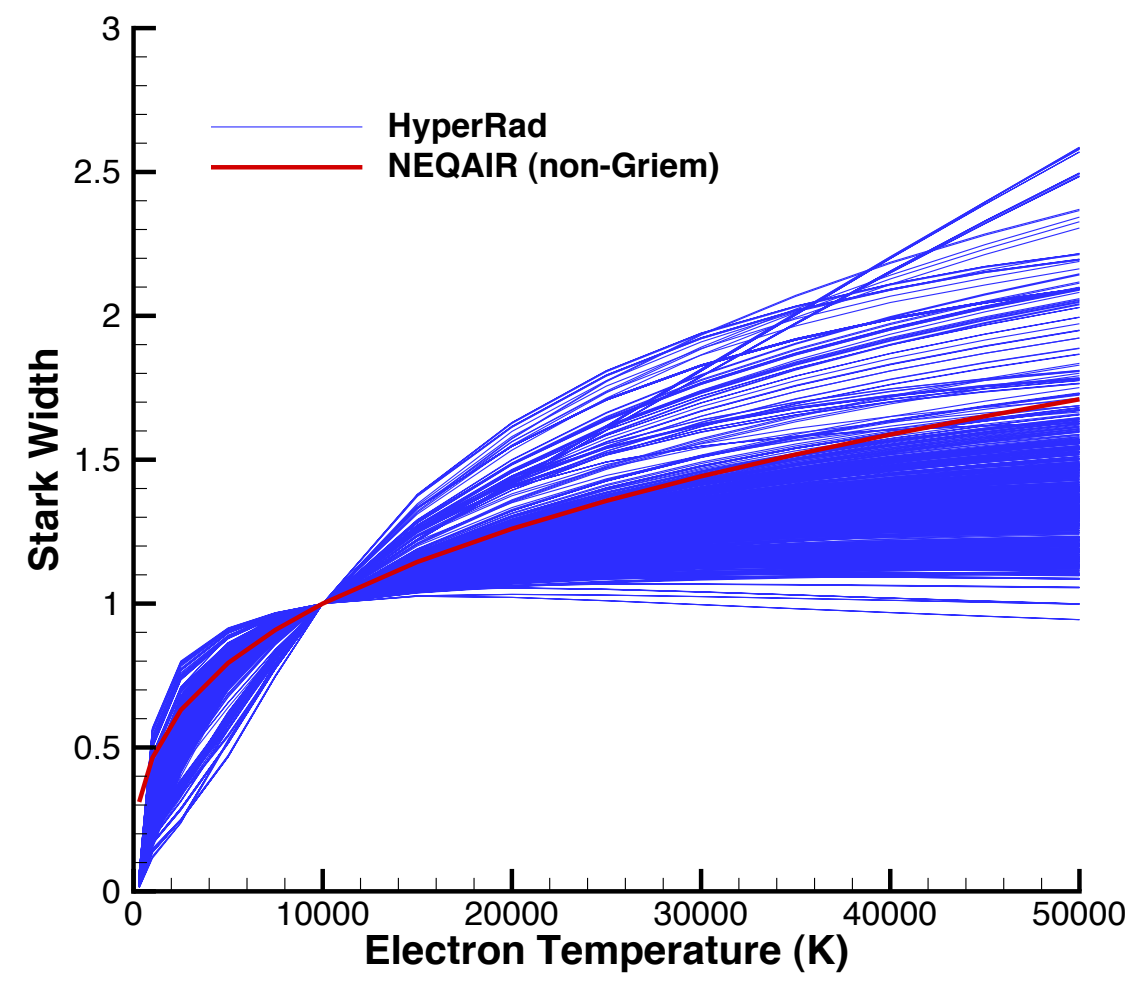

Figure 3. Temperature variation of Stark widths for atomic $\mathrm{N}$ lines

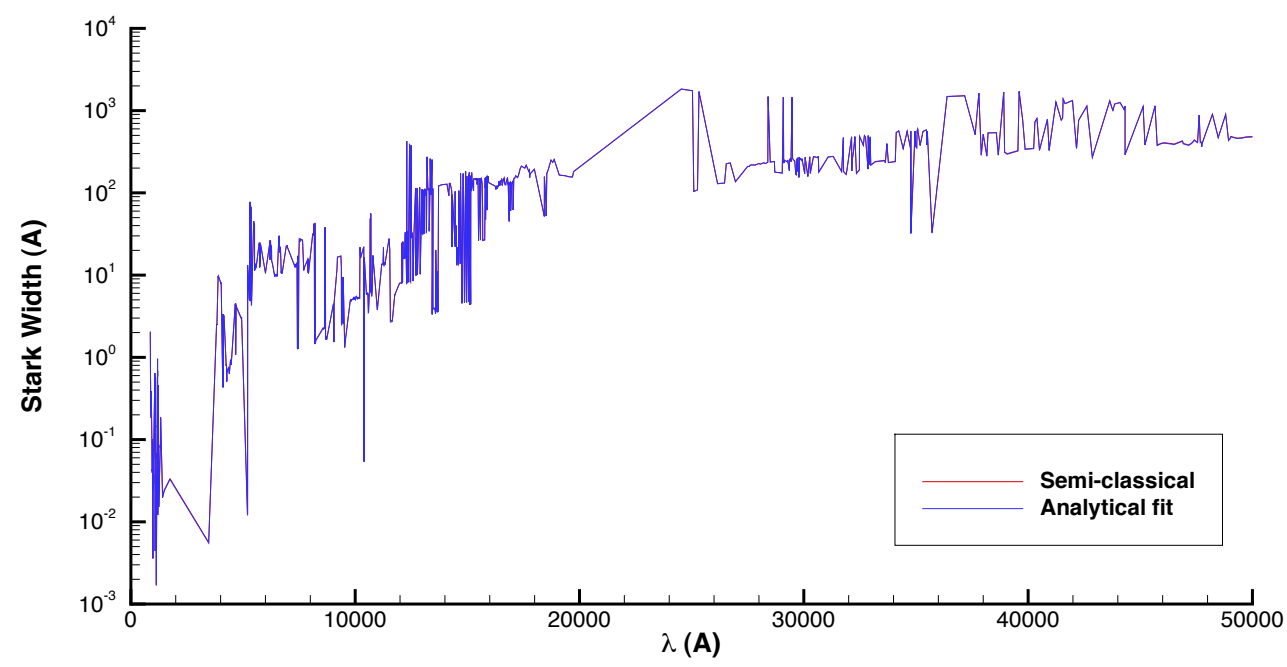

Figure 4. Comparison of semi-classical calculation and analytical fit at $12,500 \mathrm{~K}$. 